MS24-O3 New antiferromagnets

$\left[\mathrm{CuX}\left(\mathrm{pyz}_{2}\right]\left(\mathrm{BF}_{4}\right)\right.$ with $\mathrm{X}=\mathrm{Cl}$ and $\mathrm{Br}$.

Mariusz Kubus ${ }^{1,2}$, Arianna Lanza ${ }^{2}$, Nicola Casati ${ }^{3}$, Piero Macchi ${ }^{2}$, Lukas Keller ${ }^{1}$, Christoph Fiolka ${ }^{2}$, Jürg Schefer ${ }^{1}$, Christian Rüegg ${ }^{1}$, Karl Krämer ${ }^{2}$

1. Laboratory for Neutron Scattering and Imaging, Paul Scherrer Institute, $\mathrm{CH}-5232$ Villigen PSI, Switzerland

2. Department of Chemistry and Biochemistry, University of Bern, Freiestrasse 3, CH-3012 Bern, Switzerland

3. Laboratory for Synchrotron Radiation - Condensed Matter, Paul

Scherrer Institute, CH-5232 Villigen PSI, Switzerland

email: mariusz.kubus@psi.ch

The structures of new compound $\left[\mathrm{CuX}(\mathrm{pyz})_{2}\right]\left(\mathrm{BF}_{4}\right)$ with $\mathrm{X}=\mathrm{Cl}^{-}$and $\mathrm{Br}^{-}$and $\mathrm{pyz}=$ pyrazine were determined by single crystal X-ray diffraction. These tetragonal compounds crystallize in space group $P 4 / \mathrm{nbm}$. They are built from $\left[\mathrm{Cu}(\mathrm{pyz})_{2}\right]^{2+}$ layers which are connected by $\mathrm{X}^{-}$ ions along the $c$-axis. Charge is compensated by $\mathrm{BF}_{4}^{-}$ions in the voids of the $3 \mathrm{D}$ coordination compound. The antiferromagnetic interactions between the $\mathrm{Cu}^{2+}$ ions are mainly two-dimensional (2D) located within the $\left[\mathrm{Cu}(\mathrm{pyz})_{2}\right]^{2+}$ layers. This results in a broad maximum of the magnetic susceptibility around $9 \mathrm{~K}$. Towards lower temperature a kink is observed at $4 \mathrm{~K}$ which indicates long-range $3 \mathrm{D}$ magnetic order. The magnetic unit cell is doubled along the $c$-axis $(k=0,0,1 / 2)$ and the ordered magnetic moment amounts to $\mu_{x}=0.76(8) \mu_{B} / \mathrm{Cu}^{2+}$ at 1.5 $\mathrm{K}$. The moments are antiferromagnetically coupled along the $b$ - and $c$-axes. Long-range $3 \mathrm{D}$ magnetic order is observed below $T_{N}=3.9(1) \mathrm{K}$. A fit of a $2 \mathrm{D}$ Heisenberg model to the magnetic susceptibility data results in $J_{\|}=$ $9.6 \mathrm{~K}$.

Keywords: 2D antiferromagnet, copper, pyrazine, DMC, XRD, ESR
MS24-04 Structural and magnetic properties of the low dimensional fluoride

$$
\beta-\mathrm{FeF}_{3} .3 \mathrm{H}_{2} \mathrm{O}
$$

Stjepan Prugovečki ${ }^{1}$, Gwilherm Nenert ${ }^{1}$, Oscar Fabelo ${ }^{2}$, Kerstin Forsberg ${ }^{3}$, Claire V. Colin ${ }^{4}$, Juan Rodriguez-Carvajal ${ }^{2}$

1. PANalytical B.V., Twentepoort Oost 26, 7609 RG Almelo

2. Institut Laue Langevin, 71 Avenue des Martyrs, 38000 Grenoble, France

3. School of Chemical Science and Engineering, Royal Institute of Technology, Teknikringen 42, SE 10044 Stockhlom, Sweden

4. Institut Néel, 25 rue des martyrs, BP 166, 38042 Grenoble, France

email: stjepan.prugovecki@panalytical.com

The anisotropy inherent in low-dimensional (1-D) solid-state compounds leads to a variety of interesting magnetic, electronic, and optical properties, with applications including single-chain magnets for data storage,(1) multiferroics for bifunctional materials $(2,3)$ and nonlinear optical materials for second harmonic generation $(4,5)$. Certain types of 1-D materials containing isolated chains exhibit nearly ideal magnetic properties, acting as experimental models for Ising and Heisenberg spin chains, furthering our understanding of magnetic exchange in highly correlated systems (6). One of the strategies for building these 1-D magnetic materials is to incorporate small one- or three-atom linkers between magnetic centers to facilitate exchange along the chains or networks $(7,8)$. Many of these compounds are known, but very few have been created using iron centers, and most rely on relatively large bridging ligands to separate the magnetically coupled components within the structure.We report on the structural and magnetic properties of the low-dimensional fluoride $\beta-\mathrm{FeF}_{3} .3 \mathrm{H}_{2} \mathrm{O}$ using SQUID magnetometry, $\mathrm{X}$-ray and neutron diffraction. The structure consists of 1D-chains of corner-linked $\mathrm{Fe}\left[\mathrm{F}_{4}\left(\mathrm{H}_{2} \mathrm{O}\right)_{2}\right]$ octahedra running parallel to [001] and isolated water molecules. A dense network of hydrogen bonds strongly connects the Fe-F chains. The structural formula is $\mathrm{FeF}\left[\mathrm{F}_{05}\left(\mathrm{H}_{2} \mathrm{O}\right)_{05}\right] 4 . \mathrm{H}_{2} \mathrm{O}$. This material exhibits a very pronounced $1 \mathrm{D}$ character with a very broad maximum around $150 \mathrm{~K}$ in the magnetic susceptibility. Below $\mathrm{TN}=20 \mathrm{~K}$, long range magnetic order appears characterized by $\mathrm{k}=\left(\begin{array}{lll}0 & 0 & 1 / 2\end{array}\right)$. From magnetic susceptibility, the intrachain magnetic coupling is estimated to be $18 \mathrm{~K}$, while the interchain magnetic interaction is estimated to be about $3 \mathrm{~K}$. We discuss this non negligible interchain coupling in light of the crystal structure of this material.

(1) Zhang, W.-X.; et al., RSC Adv. 2013, 3, 3772-3798.

(2) Xu, G. C.; et al. J. Am. Chem. Soc. 2010, 132, 9588-9590.

(3) Xu, G. C.; et al. J. Am. Chem. Soc. 2011, 133, 14948-14951.

(4) Kandasamy, et al. Cryst. Growth Des. 2007, 7, $183-186$.

(5) Anbuchezhiyan, M. et al. Mater. Res. Bull. 2010, 45, 897-904.

(6) Coulon, C.; et al. R. In Single-Molecule Magnets and Related Phenomena; Winpenny, R., Ed.; Springer-Verlag: Berlin, Germany, 2006

(7) Wang, X.-Y.; et al. Chem. Commun. 2008, 281-294. 
(8) Sun, H.-L.; Wang, et al. Coord. Chem. Rev. 2010, 254, 1081-1100.(9) G. Nénert, et al., Dalton Transactions $44(31), 14130-14138$

Keywords: 1-D magnetic materials , X-ray diffraction, neutron diffraction, interchain coupling

\section{MS24-05 Synthesis and Characterization of Asymmertric Tetranuclear Nickel Chains without Disordered Ligand Phenomenon in Crystallography}

Lien-Hung Tsou ${ }^{1}$, Marc Sigrist ${ }^{2}$, Ming-Hsi Chiang ${ }^{2}$, Er-Chien Horng $^{1}$, Chun-hsien Chen ${ }^{1}$, Gene-Hsiang Lee ${ }^{1}$, Shie-Ming Peng ${ }^{1,2}$

1. Department of Chemistry, National Taiwan University, No. 1, Sec. 4, Roosevelt Rd., Taipei, Taiwan (R.O.C.)

2. Institute of Chemistry, Academia Sinica, 128 Academia Road, Section 2, Nankang, Taipei, Taiwan (R.O.C.)

email: d01223123@ntu.edu.tw

The new ligand,

2-(2-(5-phenylpyridyl)amino)-1,8-naphthyridine

(Hphpyany), was synthesized by the reaction of 2-chloro-1,8-naphthyridine with 2-amino-5-phenylpyridine in the presence of potassium tert-butoxide under palladium(0)-catalyzed condition. The linear tetranickel metal complexes, $\left[\mathrm{Ni}_{4}\right.$ (phpyany) $\left.{ }_{4}(\mathrm{Cl})_{2}\right]\left(\mathrm{CF}_{3} \mathrm{SO}_{3}\right) \quad \mathbf{1}$ $\left[\mathrm{Ni}_{4}{ }_{4}\right.$ (phpyany) $\left.{ }_{4}(\mathrm{Cl})_{2}\right]\left(\mathrm{BF}^{3}\right)_{2} \mathbf{2}$,

$\left[\mathrm{Ni}_{4}^{4}\right.$ (phpyany) $\left.{ }_{4}(\mathrm{NCS})_{2}\right]\left(\mathrm{ClO}_{4}\right) \quad \mathbf{3}$ and $\left[\mathrm{Ni}_{4}^{4}\right.$ (phpyany) $\left.{ }_{4}(\mathrm{NCS})_{2}^{2}\right]\left(\mathrm{CF}_{3} \mathrm{SO}_{3}\right)_{2} 4$ were synthesized and have been crystallographically characterized. All of the complexes consist of four phpyany- ligands, wrapped around a linear tetranickel core, in the same orientation. The remarkably short Ni-Ni distances (ca. $2.33 \AA$ ) for $\mathbf{1}$ and $\mathbf{3}$ indicate partial metal-metal bonding, which can be viewed as both complexes containing one mixed-valence $\mathrm{Ni}_{2}{ }^{3+}$ unit. Magnetic susceptibility measurements reveal that the $\mathrm{Ni}_{4}{ }^{7+}$ complexes exhibit antiferromagnetic interactions $\left(J=-42 \mathrm{~cm}^{-1}\right.$ for 1 and $-46 \mathrm{~cm}^{-1}$ for 3$)$ between the $\mathrm{Ni}^{2+}$ and the $\mathrm{Ni}^{3+}$ units, while the $\mathrm{Ni}_{4}{ }^{8+}$ complexes $\mathbf{2}$ and $\mathbf{4}$ exhibit antiferromagnetic interactions $\left(J=-33 \mathrm{~cm}^{-1}\right.$ for 2 and $-35 \mathrm{~cm}^{-1}$ for 4$)$ between the two terminal $\mathrm{Ni}^{2+}$ ions. The results of the cyclic voltammetry indicate the presence two reversible redox couples at $\mathrm{E}_{1 / 2}^{(1)}=0.07 \mathrm{~V}, \mathrm{E}_{1 / 2}^{(2)}=-0.80 \mathrm{~V}$ for 1 , and at $\mathrm{E}_{1 / 2}(1)=0.12$ $\mathrm{V}, \mathrm{E}_{1 / 2}=-0.74 \mathrm{~V}$ for 3 . The products of the oxidation process $E_{1 / 2}{ }^{(1)}$ of $\mathbf{1}$ and $\mathbf{3}$ are the corresponding oxidized species $\mathbf{2}$ and $\mathbf{4}$, respectively. The value of conductance is $9.39( \pm 0.301) \times 10^{-4} G_{0}$ and the value of resistance is 13.7 $( \pm 4.4) \mathrm{M} \Omega$ for 4 were measured by means of the STM break-junction. This represents the first conductance measurement of a linear tetranickel chain.

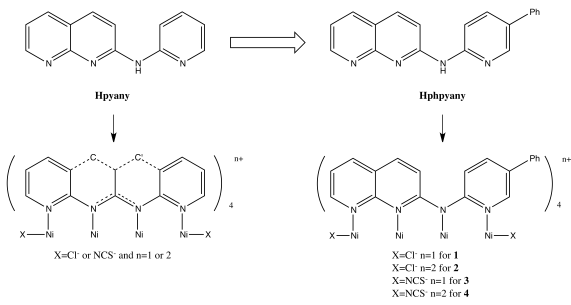

Figure 1. Crystallographic disordered ligand on $\mathrm{C}$ and C' (left bottom) and schematic diagram for complexes 1,2,3 and 4 (right bottom).

Keywords: Metal-metal interactions $\square$, Nitrogen ligands, Electrochemistry, Magnetic properties, Single molecular conductance 